\title{
The Strategic Clinical Model In Psychotherapy: Theoretical And Practical Profiles
}

\section{Giulio Perrotta}

Director of the Department of Criminal and Investigative Psychology UNIFEDER, Italy.

Corresponding Author: Giulio Perrotta, Director of the Department of Criminal and Investigative Psychology UNIFEDER, Italy. E-mail: giuliosr1984@hotmail.it

Received date: January 30, 2020; Accepted date: February 02, 2020; Published date: February 06, 2020

Citation: Giulio Perrotta (2020), j Addi Adol Beh 3(1) The Strategic Clinical Model In Psychotherapy: Theoretical And Practical Profiles Doi: 10.31579-007/2688-7517/016

Copyright: (02020 Giulio Perrotta This is an open-access article distributed under the terms of The Creative Commons. Attribution License, which permits unrestricted use, distribution, and reproduction in any medium, provided the original author and source are credited.

\section{Abstract:}

This work deals with the analysis of the theoretical and practical profiles of the strategic clinical model in psychotherapy, starting from the examination of the historical and evolutionary contents of this particular model, passing through the main theories and techniques used in the clinic.

\section{Keywords: psychotherapy; theoretical and practical profiles}

\section{Introduction. Historical contexts and epistemiological profiles}

The strategic model originates from the systemic psychological current, which refers to that complex of hypotheses and researches able to overcome the perspective centered exclusively on the individual typical of traditional psychology. It is a current that refers to von Bertalanffy's "general systems theory" (1901-1972) and cybernetics for the study of psychological communication. The main authors who founded this orientation, starting in the late 1950s, were all members of the Mental Research Institute in Palo Alto (California, United States of America), including the constructivists Watzlawick (1921- 2007) and Bateson (1904-1980), with their theories on language and groups, and inspirers with M. Erikson (1901-1980), the true father of the strategic current. [1]

The epistemological basis [2] of the evolved model is therefore the:

1) the "radical constructivism" by von Glasersfeld and von Foerster. It is an unconventional approach to the problem of knowledge and knowing. It starts from the assumption that knowledge, regardless of how it is defined, is in people's heads, and that the thinking subject has no alternative: he can only build what he knows based on his own experience. What we understand from experience constitutes the only world in which we know we live. The fundamental principles of its radical constructivism are proposed in four points: a) knowledge is not received passively either through the senses or through communication; b) knowledge is actively constructed by the "knowing" subject; c) the function of knowledge is adaptive, in the biological sense of the term, and tends towards adaptation or "viability"; d) knowledge serves the organization of the experiential world of the subject, not the discovery of an ontologically objective reality. The strategic approach therefore does not present itself as an absolute truth but as a model that offers different truths according to the different realities, as the perceived reality depends on multiple conditioning factors that determine a never objective reality. This is why there is no single reality, unique, unchanging but many realities determined by the various relationships between us and the rest of the world. Radical constructivism is therefore interested in the "how" we know reality and live it and less in the "why" (albeit important to fully investigate each profile). What people call "reality" is the result of communication between us. Reality is therefore the result of how we communicate about what is around us. Communication becomes a tool to organize actions, through communication, recognizing the ways in which people build their own reality (perceptive / reactive system) and find the resources to reshape the dysfunctional reality and identify the parts of themselves to be integrated. Radical constructivism teaches us how there is no truth or one real world. Accepting that there are different versions of reality and the world, as many as there are people, allows the therapist to always be on the patient's side, an active builder of his own reality, his beliefs and his values.

2) the "systems theory" by von Bertalanffy, Luhmann, Crozier, Friedberg, Homans, Blau and Emerson. The general theory of systems uses the mathematical concept of function (interdependence relationship between different variables) on the basis of which it examines the relationships that are actually established between the different elements of the considered system. The term system refers to a complex reality whose elements interact with each other, according to a circularity model according to which each element conditions the other and is in turn conditioned by it. The meaning of each individual element should therefore not be sought in the element 
itself, but in the system of relations in which it is inserted. The system should therefore not be understood as something that exists in reality, but rather as a theoretical elaboration, on the basis of which it is possible to account for certain phenomena. The consideration of the phenomena in their reciprocal relationship makes the systems not something static, but in constant evolution (or involution) dynamic. It should be noted that this dynamic is particularly present in systems in which relations with the surrounding environment are more frequent (open systems). The new instances that gradually arise in the environment give rise to dynamic variations that tend to bring the whole to a situation of new equilibrium. Since social systems are constituted on the basis of a shared sense, the analysis of communicative processes is essential as part of social research itself.

3) the "pragmatics of communication" by Watzlawick, Beavin and Jackson. Each communication process between human beings has two distinct dimensions, namely the content (what the words say) and the relationship (what the speakers let us understand, both verbally and otherwise, on the quality of the relationship between them). In particular, the most eminent author published in 1971 "Pragmatics of human communication. Study of interactive models, of pathologies and paradoxes", going so far as to argue that" neuroses, psychoses and in general forms of psychopathology do not arise in the isolated individual, but in the type of pathological interaction that occurs between individuals ". The interaction between people who communicate and the influence exerted on them by culture, through the signs and symbols to which meanings are attributed, was therefore fundamental. This theoretical elaboration generates five axioms: a) one cannot fail to communicate. Even silence, for example, is communication; b) each communication has one aspect of content and one of relationship, the second defines the first: as happens with the differences that can give the tone of voice, for example, the same word can be defined differently by different ways of relating; c) the nature of a relationship depends on the punctuation of the communication sequences between the communicants and each communicative action influences and is influenced by the verbal and non-verbal behavior of the interlocutor; d) human beings communicate both with the verbal (numeric) and with the non-verbal (analogical) form, therefore the language, in "digital", generally concerns the aspect of content. Non-verbal modalities, on the other hand, generally concern the relationship aspect; e) all communication exchanges are symmetric or complementary: they are symmetric between people who have similar roles; complementary between people who do not have the same role and / or the same power.

4) the "modern game theory" by von Neumann and Morgenstern. Game theory is a mathematical theory that serves to describe the rational choices that players make when they are in a situation where they have to interact strategically, that is, when a player can influence the behavior / result of another player. Game theory is based on mathematical models, a simplified representation of reality to understand how interested parties behave based on the information they have to develop a strategy. These analyzes allow us to understand, and to some extent predict and condition, the evolution of a process such as a trivial chess game or the complex functioning of the stock market. Game theory has a distant origin that has its roots in 1654 in a correspondence of Pascal and de Fermat. The modern birth, however, can be traced back to the release of the book "Theory of games and economic behavior" by von Neumann and Morgenstern in 1944. The one who during the twentieth century made the greatest contribution to this fascinating theory is Nash, American mathematician, to whom the film starring Crowe is dedicated. It is essentially a theory that attempts to define and give a concrete explanation to the behavior of people when they are in a situation that can lead to the division of an object of desire or to the latter's victory by one. Before hypothesizing a situation to understand in detail what we are talking about, we must specify some assumptions that cannot be missing in order to apply the theory. Those who participate in the "game" must play to win and maximize their winnings. The situation requires a finite number of decisions that can be taken by the participants, each decision made has positive or negative (non-neutral) implications, the game can be cooperative or non-cooperative. The best strategy, to be implemented in situations such as poker, is to minimize the maximum possible loss, everything is explained in the "minimax theorem": you need to define and imagine the worst possible scenario.

The strategic logic [1] of Watzlawick, Elster, Da Costa and Nardone is based on these theoretical foundations. The short strategic approach to therapy is evidence based (Szapocznik, Castelnuovo, Gibson, Lock, Nardone, Salvini, Robin) and is recognized as best practice for some important psychopathologies: obsessive compulsive disorder, binge eating, juvenile anorexia, panic attacks, family violence and antisocial behavior. In particular, the model formulated by Watzlawick (Brief strategic therapy) and evolved by Nardone (Giorgio Nardone's Model), as well as being empirically and scientifically validated (Nardone, Gibson, Nardone, Salvini, Castelnuovo) for almost thirty years, has led, as evidenced by the numerous publications resulting from this, to the formulation of advanced short therapy protocols, composed of innovative techniques built ad hoc to unlock the particular types of persistence of the most important psychic and behavioral pathologies (Nardone, Balbi). This model has four specific phases: knowledge of the problem and attempted solutions, definition of the objectives to be achieved, behavioral prescriptions and specific techniques and strategies, conclusion of the therapy. The intervention never exceeds twenty sessions (even if often excellent results are obtained even with half the sessions), including the three follow-up sessions at three, six and twelve months.

\section{The main characteristics of the theoretical model}

The theoretical framework of strategic psychotherapy [1] is based on specific epistemological assumptions as highlighted so far. These assumptions refer briefly to:

a) functioning of the human mind, understood as an organized structure on a "system of interdependent relationships between oneself - others - world", where man is at the center of the systemic relationships between all the components, according to a scheme of first (objective 
reality) and second (subjective) order, and no longer linear but circular (the search for a first cause (linear logic) fails because the phenomenon follows a logic of circular causality, and on three levels of functioning of the mind (visual, auditory and kinesthesic or emotional, which also includes all the other senses). In the same way, in all human relationships we influence each other with cybernetic and constructivist logics);

b) use by the subject of his personal "perceptive-reactive system", understood as the set of ways in which each of us, in a subjective way, perceives reality, attributes a certain meaning to it and reacts to it. Through the experiences of interaction (with oneself, others, and the context), the mind constructs the criteria and ways in which to interpret reality, that is, its habits to perceive-react. Our reactive perceptive system works as a filter that selects the meanings to be given to things, as a frame that frames a phenomenon interpreting it in one sense or another, according to its own criteria (emotional, motivational, logical, values and according to the states Of the mind). The individual perceptive-reactive system is the result of interaction with the environment and does not depend on genetic factors.

c) elaboration, by the subject, of "attempted dysfunctional solutions" to the problems encountered, that is what we do to solve a problem is often exactly what keeps it or makes it worse. Subjective perceptions and reactions to reality produce (even relatively quickly) habits of thinking, behaving, reacting and interacting in a certain way. These habits, once established, tend to be repeated (due to a mental economy), even when they are no longer suitable for the situation and even if they cause discomfort. They are then called "attempted solutions" because they are only unsuccessful attempts to resolve. They are redundant, that is, they tend to repeat and extend to contexts other than those in which they were built. Two examples of an attempted 'avoidance' solution. First: in the case of panic attacks, the attempted solution to avoid the panic site reinforces the belief that some places are dangerous. Thus avoidance extends to other places or different situations, becoming an 'attempted' solution, increasingly pervasive and thus building an increasingly disabling problem. Second example: in a couple the attempted solution of 'avoiding conflicts' leads to a constant fear of making mistakes, to do / speak less and less and to a progressive closure. This is then interpreted as a danger to be feared (and therefore to a greater fear of making a mistake) or as a failure (and therefore will produce forced attempts to interact, smiles and phrases that are not spontaneous and credible) or a resignation not to communicate, or yet a continuous suspicion towards the other and a greater closure. These solutions, therefore, in their dysfunctional redundancy, instead of promoting the extinction of the problems, preserve and aggravate them.

With these premises, it is clear that strategic therapeutic therapy is aimed at the solution that opens the lock, not seeking so much the "why" but the "how", since strategic interventions act to produce changes not on people themselves but on their way to interact with each other and the perceived reality and in order to therefore achieve a change of perspective and the change of maladaptive behavior into one more functional to the well- being of the subject, strategic psychotherapy, in line with the epistemological model of radical constructivism, starts from the belief that to change a problematic situation, one must first change the action and then the thinking, or rather the observation point or 'frame' of reality. So we certainly do not want to deny the influence of thought and cognition on acting but it is argued that change must pass first from the phase of concrete experience and by itself become cognitive baggage. Only through the preliminary experimentation of a corrective emotional experience (Alexander) can changes in the dysfunctional perceptivereactive system be produced, which must then be strengthened and maintained over time with learning and made evident in the evaluation of the subject. The strategic intervention is therefore structured on the methodological principle of "Research-action" or "Researchintervention", theorized by the psychologist Lewin in 1946, which, by connecting theory and practice, derives from the functional knowledge of a problem starting from modified attempts to modify with specific interventions the problem itself: this perspective is based on the conception for which it is the solution that explains the problem and not the other way around. [1]

Other peculiarities of the strategic model are; the "brevity of the therapy" (based on the problem encountered, it is possible to complete the course even in one session, in other cases instead in 6-8 sessions it is the best option); the "the exact identification of the objectives to be achieved" (what we are trying to achieve to break the dysfunctionality that supports the cause of suffering); the "suppression of the belief that the pathological state is irreversible or serious" (through the use of a series of techniques aimed at making the patient live corrective emotional experiences intended as experiences that nullify the rigidity of the theoretical position previously assumed by the patient towards the his state of health); the use of "specific targeted protocols" for the management and treatment of certain morbid pathological conditions (there are over forty fo them, for example for the panic attack, capable of solving the problem in $85-90 \%$ of cases, but also for and phobias, disorders food, sexual, mood and addictions). [1]

\section{The main techniques used in the clinic}

There are several techniques that are now part of the strategic therapist's clinical background. So far we have understood that the three pillarsof strategic psychotherapy are certainly communication, relationship and specific techniques. [1]

In particular, among the most incisive and functional techniques [3]:

1) the guided fantasy. It is a technique aimed at "structuring an imaginary topic between the subject and the operator, in which one tries to reach a point from which the person can observe, directly or as an allegory, the knots of emotional conflict. The topic of fantasies will be developed by both: the therapist stimulates, encourages, persuades without forcing the person, seated and with their eyes closed, to recount the actions that develop in his inner world;

2) the direct, indirect and paradoxical prescriptions. The "direct prescriptions" are clear indications of actions to be performed. For 
example, a parent who constantly motivates the child to study, producing refusal in him, may be instructed to temporarily stop his vain efforts, so that the child reduces his or her refusal attitude. Or, to those who suffer from problems of an anxious nature, and who constantly talk about his problem without realizing that by doing this he feeds his anxieties, one can ask to limit communications on the problem, in order to avoid his attempted dysfunctional solution. Of course, it is clear how these types of prescriptions can be used almost exclusively with very collaborative people, that is, who want and can put into practice what is required. Or they can be used in advanced stages of therapy, when the problem is now almost totally solved and the person must be asked to do certain tasks useful for maintaining the results achieved. "Indirect prescriptions" are instead the kind of injunctions of behavior that mask their true goal: the patient is prescribed to do something that will (also) produce an effect different from what he explicitly expected. For those who suffer from panic attacks, for example, it may be prescribed to keep a diary of their attacks, to be filled in at the exact moment of the onset of each of them, so that they can be monitored: the secondary purpose, however, will be to move the attention of the person on a simple but demanding task, so that he avoids monitoring his own bodily functions instead. To a man who thought himself incapable of crossing the street, Erickson absently asked to do him a favor: since he was in a wheelchair, he asked him to post a letter for him in the nearby box. To do so, the man did not realize that he crossed the road twice except when he returned from Erickson. This produced a first emotional corrective change experience. Watzlawick spoke about "planned random events": the therapist prescribes the patient to make an apparently neutral action (post a letter) which will produce an apparently random effect (being able to cross the street) with therapeutic effects. These types of prescriptions are very useful for circumventing resistance, especially where this is given by limits related to self-perceptions. Finally we come to the "paradoxical prescription" or, in a more clinical sense, the prescription of the symptom which, as Watzlawick, Weakland and Fisch claim "is undoubtedly the most effective and elegant form we know of solving problems". The problems, therefore, also include (but not only) the symptomatic conditions. In this sense, the paradoxical prescription, already studied by the psychoanalyst Frankl (1960), requires to ask the person (or to put them in conditions) to implement exactly the behavior they want to be freed: in this way the voluntary execution cancels it the aspect of unstoppable spontaneity, putting it under conscious control and quickly canceling its presence. Among the well-known and most used examples of paradoxical prescriptions, it is possible to think of the patient victim of obsessive thoughts who are asked, in certain specifically agreed spaces, to think voluntarily precisely about those thoughts; or, to a sleepless person who cannot sleep, it can be indicated that he remains awake at all costs to perform an unpleasant task, such as waxing the parquet; a boy tormented by the fear of being blamed is asked to intentionally ridicule himself several times with simple and naive actions. The strength of the paradoxical prescriptions is such that almost always the problem is released in a few sessions and begins to reduce its intensity and frequency, until it disappears. Of course, all prescriptions must be tailored, contextualized to the patient's reality and adapted to his situation.

3) The use of strategic dialogue (logical and analog/emotional), consisting of metaphors, paradoxes, empathize with your demon, strategic questions (with the answer limited to only two possible options), restructuring paraphrase (reformulating the patient's responses to postpone the outcome of his reworking process, to ensure the best reconstruction, even with suggestive evocations, further circumventing the resistance to change), emphasis or devalutazion of specific events, logbooks and constructive manipulations, aimed at helping the patient to re-elaborate his interpretation of reality better and more centrally. Furthermore, using irony as a communication technique is often functional to relieve tension and help the patient and relax in a difficult moment. Again, borrowing from the Gestalt school, the empty chair technique can be effective in releasing internal emotional tensions and facing a person or situation that determines anxiety and emotional tensions.

4) Hypnosis. It is a psychosomatic phenomenon that can be caused by a suggestion due to an image or sound that the subject perceives intensely. Hypnotism has ancient historical roots, since the times of the Chinese, Egyptians, Indians, Jews, Greeks and Romans and is a theme transversal to many psychological currents that use this therapeutic tool. The modern historical epochs can be summarized in these three periods: a) "mystical": with Paracelsus (1490-1541), in 1530, a great scholar of astrology and occultism. The 17th century will bring the Enlightenment with it; b) "magnetic": with Mesmer (1734-1815), hypnosis passes from the esoteric world to the scientific (psycho-physiological) world; however, however, his beliefs about "animal magnetism" and man's powers through the use of his hands will place him in antithesis with the scientific system of the time. $\mathrm{He}$ came to understand the influence of the Moon on the tides and on the female menstruation (which follows a lunar cycle), but his idea of healing hysterical blindness will make him a heretic, cut short by the Academy of France for his "suggestive manipulation" ; c) "psychological": with Braid (1785-1860), in the eighteenth century, Mesmer's theories were revised, becoming from "animal magnetism" to "hypnosis or neuro-hypnotism", showing that the phenomenon was only a suggestibility, due to the result between the concentration on one point and a predominant psychic cause (so-called monoideism), far from the fluid of which Mesmer spoke. Subsequent studies will lead to the demonstration that to reach the hypnotic state it will be enough just to imagine a point in one's mind and not necessarily to focus it materially, passing in fact from the concept of "suggestibility" to "suggestion". Prof. Bernheim (1837-1919) and Liébeault (18231904) were great supporters of hypnotic techniques (from the Nancy School), and considered hypnosis as a psychological phenomenon consisting of a state of activated and increased suggestibility; Charcot (1825-1893) (of the Salpétriére School) also approached hypnosis but only in 1878 . For the latter, hypnosis was an instrument of experimental investigation and not a therapeutic technique for a disease that was at the time classified as "hysteria"; therefore, it was usable only with these subjects and not with all of them, as the 
antagonists of his school claimed. Again, Janet (1859-1947) laid the theoretical foundations for the studies of Freud and Jung, arguing that hypnosis was nothing more than a form of "dissociation", or a momentary transformation of an individual's mental state, artificially induced by another person is sufficient to cause dissociation; therefore, a sleepwalking influence determined by the relationship between hypnotist and hypnotized. For S. Freud (1856-1939), he preferred the theory of the interpretation of dreams and free associations to the physiological state of hypnosis. For him, hypnosis was a regressive state maintained by the archaic relationship with the hypnotist, according to a pattern of credulity and dependence on the latter; Ferenczi was of the same thesis. In Russia, Pavlov (18491936), starting from the idea of regression, came to define the physiological concept of "hypnosis" or "hypnotic somnolence" as a process of widespread cortical inhibition with some sensitive points where it is possible to intervene through suggestion. Again, Hull (1884-1952) studied hypnosis according to the behaviorist approach, claiming that it was a state of generalized hyper-suggestibility. Still in this current, one of the leading experts was M. Erikson (19021980), father of the short strategic psychotherapeutic approach and modern user of hypnosis, not exclusively linked to the treatment of mental problems and neurosis. Thus he developed a form of hypnotherapy called "Ericksonian hypnosis", which would allow communication with the patient's unconscious. This type of hypnosis, very similar to a normal conversation, which often can also use metaphors and a persuasive and poetic language, induces, relaxing the patient, a sort of hypnotic trance in the subject, that is, a state halfway between sleep and waking. This is visible when the patient has trance indicators: half-open or closed eyes, slight states of altered consciousness (such as light "simple" hallucinations - generally sounds or colors - similar to dreams or perceptions of half-sleep), relaxation of the muscles, breathing slowed down, possible trance period amnesia, analgesia, mild sleepwalking, etc. In this way, the therapist can suggest ways of solution to the unconscious, circumventing the resistances and the repression that consciousness would oppose to change. If the patient experiences some trance signs when hearing the story or listening to the therapist's words, he is in the necessary conditions to intervene. Erickson, like Bateson, believed that everyone had their own self-built reality, and that entering into and changing the patient's perceptions (by speaking with the unconscious) was the only way to change it. The therapist must therefore adapt to the patient, the central pivot of the therapy and feeder of the disorder. The purpose of psychotherapy is in fact to allow the patient to regain possession of his own subjective resources, working on the present and not on the past; hypnosis is a reeducational technique that does not represent the source of healing that induces a psychosomatic condition of impaired consciousness, while the trance state instead allows you to more easily deconstruct dysfunctional behaviors, with the fundamental collaboration of the patient. Electrophysiological studies have recently shown that two areas of the limbic system are involved in the hypnotic state, i.e. the hippocampus (maintenance) and the amygdala (awakening).

\section{Conclusions}

Although the strategic method was already known four thousand years ago in China and two thousand and five hundred years ago in Ancient Greece, the strategic model applied in psychology finds paternity thanks to the studies of M. Erikson, who reorganizes the concepts and gives him educational dignity. Dozens of authors after him contributed to the dissemination of strategic knowledge and despite various economic speculations on specific theories and approaches, more aimed at the resolving goal of the individual problem narrated by the patient and not taking care of it in its entirety, this model (together with cognitivebehavioral and dynamic) it has obtained scientific validity for years, so much so that it can take advantage of over forty specific clinical protocols for the major psychopathologies, including mood disorders, phobias, panic attacks, sexual disorders, disorders sleep, post-traumatic stress disorder and obsessive-compulsive disorder. A very recent evolution of the short strategic model is given by the "single session therapy" by Talmon, Hoyt and Rosenbaum: a method that on the one hand manages, by itself, to lead to success in a large series of cases; on the other hand, by integrating it within its own reference model (whether it is psychotherapy or psychological counseling), it allows you to maximize its effectiveness. In fact, this means that many people experience the effects of a short therapy: in a few sessions, often even in one, they manage to achieve the goal they had set themselves. In the future, greater attention is always expected towards this model that could totally revolutionize the way of doing psychotherapy, while being careful not to focus too much on the immediate goal, leaving out the definitive functional change. [1] [2]

\section{References}

1. Perrotta G., Psicologia generale. Luxco ed., I ed., 2019.

2. Perrotta G., Psicologia dinamica. Luxco ed., I ed., 2019.

3. Perrotta G., Psicologia clinica. Luxco ed., I ed., 2019. 